\title{
Asymmetric transmission and anomalous refraction in metal nanowires metasurface
}

\author{
A. Belardini \\ alessandro.belardini@uniroma1.it \\ F. Pannone \\ G. Leahu \\ M. C. Larciprete \\ M. Centini \\ C. Sibilia \\ C. Martella

\section{Giordano \\ D. Chiappe}

\section{F. Buatier de Mongeot}

Dipartimento di Scienze di Base e Applicate per l'Ingegneria, Sapienza Università di Roma, Via A. Scarpa 14, 00161 Roma, Italy

Dipartimento di Scienze di Base e Applicate per l'Ingegneria, Sapienza Università di Roma, Via A. Scarpa 14, 00161 Roma, Italy

Dipartimento di Scienze di Base e Applicate per l'Ingegneria, Sapienza Università di Roma, Via A. Scarpa 14, 00161 Roma, Italy

Dipartimento di Scienze di Base e Applicate per l'Ingegneria, Sapienza Università di Roma, Via A. Scarpa 14, 00161 Roma, Italy

Dipartimento di Scienze di Base e Applicate per l'Ingegneria, Sapienza Università di Roma, Via A. Scarpa 14, 00161 Roma, Italy

Dipartimento di Scienze di Base e Applicate per l'Ingegneria, Sapienza Università di Roma, Via A. Scarpa 14, 00161 Roma, Italy

Dipartimento di Fisica, Università di Genova and CNISM, Via Dodecaneso 33, 16146 Genova, Italy

Dipartimento di Fisica, Università di Genova and CNISM, Via Dodecaneso 33, 16146 Genova, Italy

Dipartimento di Fisica, Università di Genova and CNISM, Via Dodecaneso 33, 16146 Genova, Italy

Dipartimento di Fisica, Università di Genova and CNISM, Via Dodecaneso 33, 16146 Genova, Italy

Here we investigated the asymmetric transmission and the anomalous refraction introduced by a metasurface of bent gold nanowires. The refraction follows the generalized Snell's law that takes into account the resonant behavior of metallic nanostructures located at the interface between two dielectrics. Measurements performed in the linear optical regime reveal a large sensitivity to the subwavelength features of the gold nanostructures.

[DOI: http://dx.doi.org/10.2971/jeos.2012.12051]

Keywords: Anomalous refraction, generalized Snell's law, metal nanowires

\section{INTRODUCTION}

Recently F. Capasso and coworkers [1] shown how it is possible to bend a ray of light by properly design resonant metallic nanostructures at the interface between two dielectrics. The metallic nanostructures in resonant condition can act as nanoantennas producing a large phase shift in the incoming light wavefront that can be of the order the wavelength [1, 2], even if the thickness of the metallic element can be very small (few tenth of nanometers). As a result the ray of light passing through two consecutive dielectric media (labeled 1 and 2 respectively) separated by the nano-patterned metasurface [3], will refract by following a generalized Snell's law [1]:

$$
\mathrm{n}_{2} \cdot \sin \left(\varphi_{2}\right)-\mathrm{n}_{1} \cdot \sin \left(\varphi_{1}\right)=\frac{\lambda_{0}}{2 \pi} \cdot \frac{d \Phi(x)}{d x}
$$

where $\mathrm{n}_{1}$ and $\mathrm{n}_{2}$ are the refractive indices of the dielectric 1 and 2 respectively, $\lambda_{0}$ is the beam wavelength in vacuum and $\Phi(x)$ represents the phase discontinuity introduced by the nanostructure at the point $x$ of the interface.
This phenomenon can be used in order to produce very thin optical elements as aberration free lenses [4], but on the other side the generalized Snell's law can be used as a powerful tool in the subwavelength characterization of nanopatterned surfaces. By varying the incidence angle $\varphi_{1}$ from Eq. (1), it is possible to retrieve information on the phase gradient $\frac{d \Phi(x)}{d x}$ introduced be the metasurface by simply measuring the refracted angle $\varphi_{2}$ of the out-coming light. Alternatively by fixing the incoming light at normal incidence $\left(\varphi_{1}=0\right)$ and by scanning the surface in the transverse direction it is possible to map the phase gradient point-by-point by using the relation

$$
\frac{\lambda_{0}}{2 \pi} \cdot \frac{d \Phi(x)}{d x}=\mathrm{n}_{2} \cdot \sin \left(\varphi_{2}\right)
$$

derived by Eq. (1) with the condition $\varphi_{1}=0$. Since the phase gradient $\frac{d \Phi(x)}{d x}$ is highly dependent on the geometrical shape of the nanoantennas, in both the previous cases important information on surface morphology can be obtained with subwavelength resolution. 


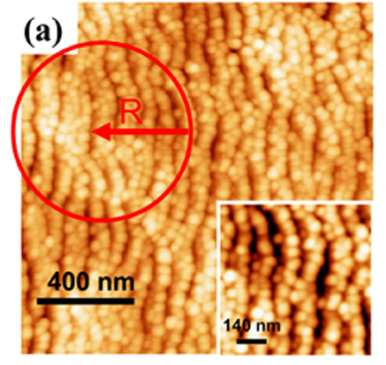

(b)

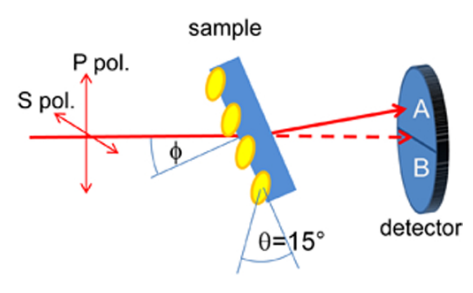

FIG. 1 (a) AFM morphology of the NWs test sample. In the inset a magnified detail show the polycrystalline gold NWs. (b) scheme of the experimental set-up and of the sample cross-section.

In this work we report the measurements in the visible spectral range of the anomalous refraction raised by a metasurface made up of self assembled bent gold nanowires (NWs) arrays with $125 \mathrm{~nm}$ of periodicity deposited onto a patterned glass slide. These measurements, synthetically shown in [5] and here extensively discussed, clearly address the dependence on the morphology of the nanowires.

\section{SAMPLE DESCRIPTION}

As described in [5], the NWs test sample has been realized on a 140 micron thick soda-lime glass substrate that was previously patterned by Ar+ ion beam sputtering. The resulting patterning (see Figure 1(a)) forms a periodic corrugations whose wavevector is mainly oriented parallel to the ion beam projection and with periodicity of about $125 \mathrm{~nm}$.

The in-plane radius of curvature $\mathrm{R}$ of the undulations follows a broad distribution centered around $400 \mathrm{~nm}$ (Figure 1(a)). In a second stage, Au was thermally evaporated under grazing incidence condition $\left(80^{\circ}\right.$ respect to the normal) and orthogonally to the ripple structures in order to localize metal agglomeration in correspondence to the convex side of the ridges. Under these conditions, a 1-D connected polycrystalline $\mathrm{Au}$ NWs is formed on top of the supporting illuminated ridges as evidenced in the inset of Figure 1(a), where a magnified image of the atomic force microscopy (AFM) topography is presented. The resulting NWs have a width of about $50 \mathrm{~nm}$, while the height amounts to about $40 \mathrm{~nm}$. The wires are laterally tilted by $\theta=15^{\circ}$ with respect the average sample surface (see Figure 1(b), where the tilt angle $\theta$ is evidenced). The optical transmission spectra obtained by using polarised light was shown in [5] and clearly confirm the excitation of localised plasmon resonances centered around a wavelength of $600 \mathrm{~nm}$ for light polarised in the transverse direction with respect to the wires, meanwhile, for light polarised in the longitudinal direction, the transmission spectra resemble those of a continuous connected $\mathrm{Au}$ film. The sample dimension is $1 \mathrm{~cm} \times 1 \mathrm{~cm}$ and it is fully patterned all over the surface and fully covered by gold nanowires except for a region of $5 \mathrm{~mm} \times 5 \mathrm{~mm}$ where the gold was removed in order to get a reference area for the forthcoming measurements.

\section{EXPERIMETAL SET-UP}

In order to study the anomalous refraction behaviour of the NWs sample a displacement measurement set-up was buildup as shown in Figure 1(b). The collimated light source is a He-Ne laser (emission mode TEM00, wavelength $\lambda_{0}=633 \mathrm{~nm}$, divergence $1.7 \mathrm{mrad}$, beam diameter $0.5 \mathrm{~mm}$ ). The wavelength of the source lies closely to the peak of the plasmonic resonance of the NWs. The polarization of the light can be set to $s$ or p polarization state. The light is then sent onto the NWs sample at an incidence angle $\varphi$. The angle is controlled by a motorized rotation stage connected to a computer. The light at the output of the sample is detected by a photodiode divided in sectors (labeled $A$ for left side sector and $B$ for right side sector in Figure 1(b)) with a diameter of $8 \mathrm{~mm}$. The detector is posed $30 \mathrm{~cm}$ far away from the sample rotation axis. The output of the sensor was analysed by a position detector circuit giving at the same time the total power

$$
P_{T}=P_{A}+P_{B},
$$

impinging on the detector and the normalised difference between the power of the light in the sector $A$ and the power of the light in sector $B$ :

$$
X=\frac{P_{A}-P_{B}}{P_{A}+P_{B}}
$$

The normalization is necessary in order to remove the effect of different light intensities, due, for example, to the different transmitted signal for $s$ and $p$ polarization state. The $P_{T}$ value is supplied in volts at the output of the circuit and is proportional to the total power by a conversion factor of $0.5 \mathrm{~mW} / \mathrm{V}$. The $X$ value is proportional to the displacement of the beam with respect to the centre of the detector and was supplied in volts at the output of the circuit. Measurements conversion from the circuit signal (in volts) to the real displacement (in $\mathrm{mm}$ ) is retrieved by a calibration measurement performed onto a glass slide of known thickness and results to be $0.05 \mathrm{~mm} / \mathrm{V}$ [5].

Since in [5] we reported exhaustively the displacement introduced at normal incidence $\left(\varphi_{1}=0\right)$, here all the displacement measurements are reported by setting the zero displacement at normal incidence, in this way it is possible to easily compare measurements obtained in different conditions.

\section{RESULTS}

In Figure 2(a) we show, as a function of the incidence angle $\phi$, the displacement $X$ and the total power $P_{T}$ measured when the light passes through the gold NWs and, as a reference, through the bare patterned glass, when the wires are mainly oriented in the vertical direction with the curvature facing the right side.

The polarization was set in order to excite the plasmonic resonance, i.e. with the polarization of the light perpendicular to the wires direction (in [5] we demonstrate that the anomalous deviation disappears when the light is polarized in a offresonance condition, i.e. with the polarization of the light parallel to the wires direction). The displacement $X$ of the glass 
(a)
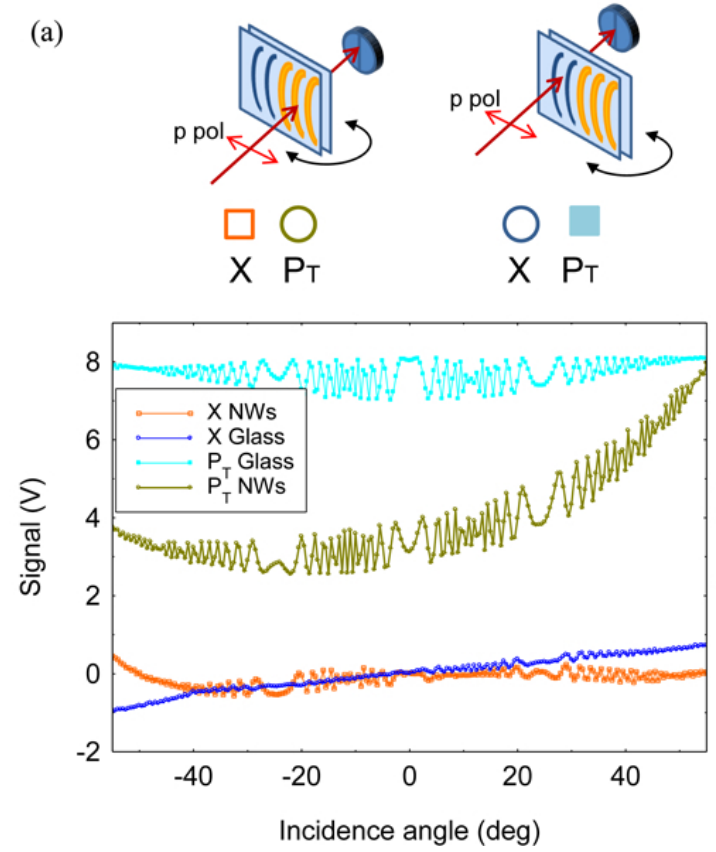

(b)
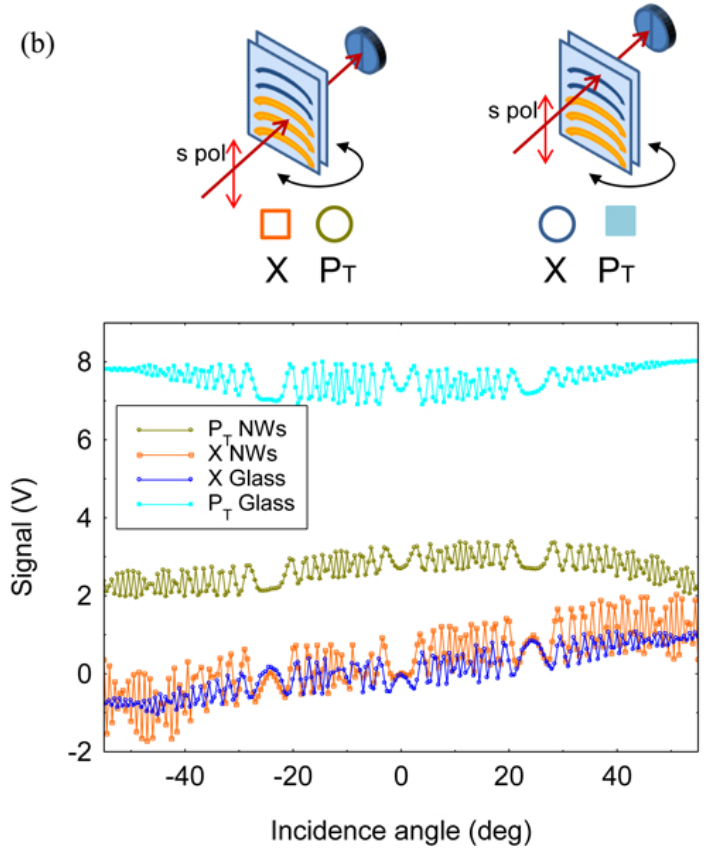

FIG. 2 Measurements of the displacement $X$ and of the transmitted power $P_{T}$ as a function of the incident angle on glass and on NWs, in the case of NWs oriented in (a) vertical direction and (b) horizontal direction. The circle arrows aside the samples schematic show the measurement rotation axis.

(blue circles) follows the standard trend of a planar glass slab (see [5]), meanwhile the gold NWs behaves anomalously (red squares). Even the total power $P_{T}$ gives interesting information, in fact the $P_{T}$ behaviour of the glass (blue squares) is perfectly symmetric while the $P_{T}$ of NWs is very asymmetric (green circles).

In [5] we shown that by reversing the orientation of the NWs, i.e. with the sample rotated by $180^{\circ}$ around the axis of the motorized rotation stage and so by entering from the substrate side, the displacement follows a reverse behaviour with respect to the starting case, but the tilt angle of the wires remains unchanged. Here, in Figure 2(b), we show the measurements when NWs are mainly oriented in the horizontal direction with the curvature facing down, so there is any predominance of the left or right side in the radius of curvature. It is very interesting to notice that the displacement $X$ introduced by the NWs (red squares) follows almost exactly the trend of the bare glass (blue circles) and no anomalous deviation is observed. This fact confirm that the displacement $X$ is sensitive to the curvature of the wires and to its orientation with respect the incidence plane. Also the total power $P_{T}$ (green circles) results almost symmetric even if the polarization is set in the on-resonance condition (s pol.).

In Figure 3 we show the measurements of the total power $P_{T}$ as a function of the incidence angle $\phi$, for different sample orientation, but with the wires mainly oriented in the vertical direction and the polarization of the light set in the on-resonance condition (p pol.). As a reference is considered the $P_{T}$ of the sample when the light is polarized in the off-resonance condition (s pol.); in this case (black triangles) the curve results to be symmetric, indicating that any asymmetry must be ascribed to the plasmonic response of the nanowires.

It is interesting to observe that if the sample is flipped (see the schematic in Figure 3 ) the $P_{T}$ changes the symmetry (green circles) with respect to the $P_{T}$ obtained when the sample is in the original orientation (red squares). In this case the change in the symmetry cannot be ascribed to the curvature of the wires, in fact, by flipping the sample, the radius of curvature lies in the same direction. It is the tilt $\theta$ of the NWs that change the sign passing from $15^{\circ}$ to $-15^{\circ}$ (see the schematic in Figure 3). If the sample is rotated by $180^{\circ}$ around the rotation axis $\phi$, the curvature of NWs change the sign of their direction (from right to left), meanwhile the tilt of NWs remains the same; in this case the $P_{T}$ (blue circles) remains with the same symmetry of the original orientation (red squares). This indicates that the $P_{T}$ is sensitive to the projection of the tilt of the NWs in the incidence plane.

\section{DISCUSSION}

These results show that the use of the deviation measurement set-up can be a sensitive method for sub-wavelength morphological analysis of nano-structured samples. Usually linear optics is used in order to retrieve information on samples with details that are in the scale of the wavelength [18] and the sensitivity can increase in the presence of geometrical resonances such as for example in the case of optical gratings [6] or photonic band-gap structures [7, 19]. In our case it is the plasmonic resonance of the metal that, even if the thickness is in the nm scale, allows a good sensitivity in the visible wavelength scale. A larger sensitivity can be achieved by using nonlinear optical phenomena such as second harmonic generation SHG [8,9], but of course short optical pulses with high intensities are needed and the measurement set-up results to be more complex. In particular SHG can be effectively used $[10,11]$ in order to detect the presence of extrinsic chirality $[12,13]$. In [14] J. B. Pendry demonstrated that optical activity [15] can be used in order to obtain anomalous refraction and in [12] N. I. Zeludhev showed that bent metallic wires 

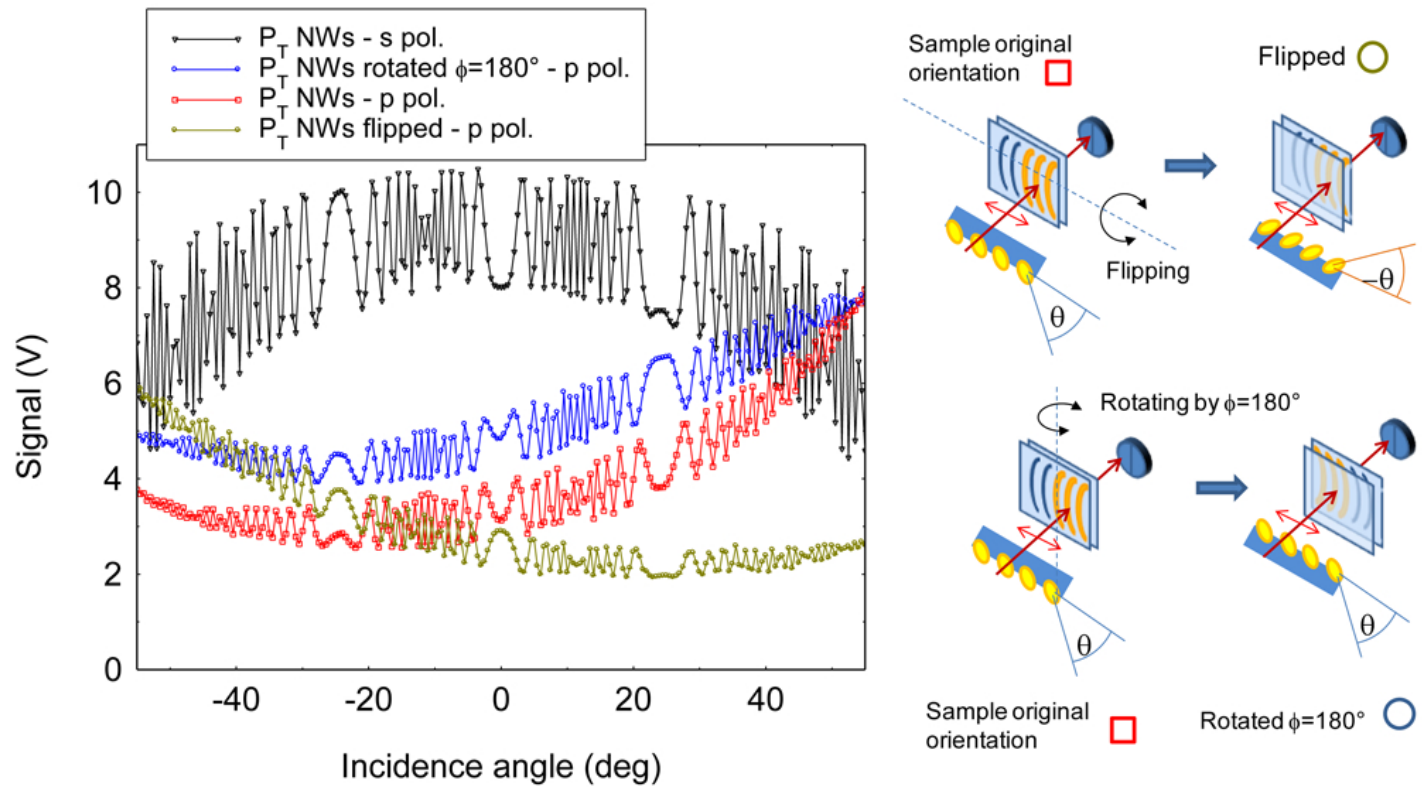

FIG. 3 Measurement of the total transmitted power $P_{T}$ as a function of the incidence angle for different geometrical orientation of the sample; with NWs which curvature faces the right side (red squares for $\mathrm{p}$ polarized light, black triangles for s polarized light), with the sample flipped (green circles) and with the sample rotated by $180^{\circ}$ (blue circles).

present extrinsic chirality and, at the same time, present very unusual refractive properties. In [16] by using circularly polarized SHG measurements we demonstrated that gold bent NWs, similar to the one here presented, give rise to evident extrinsic optical chirality that is closely linked to the curvature $R$ of the NWs, meanwhile in [17] we used linear polarised SHG measurements on the same sample and we demonstrated that the SHG signal is sensitive to the tilt $\theta$ of NWs. Here both of these issues (radius of curvature and tilt) can be investigated by using a simple linear optical set-up.

\section{CONCLUSIONS}

In conclusion here we have investigated the anomalous refraction introduced by curved gold nanowires deposited onto a glass slide. The section of the wires $(40 \mathrm{~nm})$ and their periodicity $(125 \mathrm{~nm})$ are comparably smaller than the wavelength used in the measurement $(633 \mathrm{~nm})$, nevertheless the results are very clear and different morphological aspects can be detected. We shown that the direction of the average radius of curvature of the wires can be determined by measuring the displacement of the light on a detector divided in sectors. At the same time the total amount of the transmitted intensity of the light brings information on the average tilt of the wires. These behaviour is due to the cooperative effect of geometrical aspects and plasmonic resonance. The overall structure acts like a low-cost and large-area effective metasurface. The simplicity of the experimental set-up and his high sensitivity can be used in order to investigate different kind of nanostructured materials.

\section{ACKNOWLEDGEMENTS}

This work was partially financed by PRIN-MIUR 2008J858Y7, by MARINE project, by Fondazione Carige and by ENEA under project MSE.

\section{References}

[1] N. Yu, P. Genevet, M. A. Kats, F. Aieta, J.-P. Tetienne, F. Capasso, and Z. Gaburro, "Light Propagation with Phase Discontinuities: Ceneralized Laws of Reflection and Refraction," Science 334, 333-337 (2011).

[2] X. Ni, N. K. Emani, A. V. Kildishev, A. Boltasseva, and V. M. Shalaev, "Broadband Light Bending with Plasmonic Nanoantennas," Science 335, 427 (2012).

[3] N. Engheta, "Antenna-Guided Light," Science 334, 317-318 (2011).

[4] F. Aieta, P. Genevet, M. A. Kats, N. Yu, R. Blanchard, Z. Gaburro, and F. Capasso, "Aberration-Free Ultrathin Flat Lenses and Axicons at Telecom Wavelengths Based on Plasmonic Metasurfaces," Nano Lett. 12, 4932-4936 (2012).

[5] A. Belardini, F. Pannone, G. Leahu, M. C. Larciprete, M. Centini, C. Sibilia, C. Martella, et al., "Evidence of anomalous refraction of self-assembled curved gold nanowires," Appl. Phys. Lett. 100, 251109 (2012).

[6] A. Sinibaldi, N. Danz, E. Descrovi, P. Munzert, U. Schulz, F. Sonntagd, L. Dominici, and F. Michelotti, "Direct comparison of the performance of Bloch surface wave and surface plasmon polariton sensors," Sensor. Actuat. B 174, 292-298 (2012).

[7] A. Belardini, A. Bosco, G. Leahu, M. Centini, E. Fazio, C. Sibilia, M. Bertolotti, et al., "Femtosecond Pulses Chirping Compensation by Using 1-D Compact Multiple Defect Photonic Crystals," Appl. Phys. Lett. 89, 031111 (2006).

[8] G. D'Aguanno, M. C. Larciprete, N. Mattiucci, A. Belardini, M. J. Bloemer, E. Fazio, 0. Buganov, et al., "Experimental study of Bloch vector analysis in nonlinear, finite, dissipative systems" Phys. Rev. A 81, 013834 (2010).

[9] M. C. Larciprete, F. A. Bovino, M. Giardina, A. Belardini, M. Centini, C. Sibilia, M. Bertolotti, et al., "Mapping the nonlinear optical susceptibility by noncollinear second-harmonic generation" 0 pt. Lett. 34, 2189-2191 (2009).

[10] V. K. Valev, B. D. Clercq, X. Zheng, D. Denkova, E. J. Osley, S. Vandendriessche, A. V. Silhanek, et al., "The role of chiral local field enhancements below the resolution limit of Second Harmonic Generation microscopy," Opt. Express 20, 256-264 (2012). 
[11] E. A. Mamonov, T. V. Murzina, I. A. Kolmychek, A. I. Maydykovsky, V. K. Valev, A. V. Silhanek, T. Verbiest, et al., "Chirality in nonlinear-optical response of planar G-shaped nanostructures," Opt. Express 20, 85188523 (2012).

[12] E. Plum, X.-X. Liu, V. A. Fedotov, Y. Chen, D. P. Tsai, and N. I. Zheludev, "Metamaterials: Optical Activity without Chirality," Phys. Rev. Lett. 102, 113902 (2009).

[13] T. Verbiest, M. Kauranen, Y. Van Rompaey, and A. Persoons, “Optical Activity of Anisotropic Achiral Surfaces," Phys. Rev. Lett. 77, 1456-1459 (1996)

[14] J. B. Pendry, and A Chiral, "Route to Negative Refraction," Science 306, 1353-1355 (2004).

[15] E. Fazio, W. Ramadan, A.Belardini, A. Bosco, M. Bertolotti, A. Petris, and V. I. Vlad, " $(2+1) D$ vortex soliton-like propagation in photorefractive $\mathrm{Bi}_{12} \mathrm{SiO}_{20}$ crystals," Phys. Rev. E 67, 026611 (2003).
[16] A. Belardini, M. C. Larciprete, M. Centini, E. Fazio, C. Sibilia, D. Chiappe, C. Martella, et al., "Circular Dichroism in the Optical SecondHarmonic Emission of Curved Gold Metal Nanowires," Phys. Rev. Lett. 107, 257401 (2011).

[17] A. Belardini, M. C. Larciprete, M. Centini, E. Fazio, C. Sibilia, M. Bertolotti, A. Toma, et al., "Tailored second harmonic generation from self-organized metal nano-wires arrays" 0pt. Express 17, 3603-3609 (2009).

[18] M. Agour, P. F. Almoro, and C. Falldorf, "Investigation of smooth wave fronts using SLM-based phase retrieval and a phase diffuser," J. Europ. Opt. Soc. Rap. Public. 7, 12046 (2012).

[19] F. Xia, M. Yun, M. Liu, J. Liang, W. Kong, and Y. Wan, "Subwavelength resolution of the annular photonic crystal with negative refraction," J. Europ. Opt. Soc. Rap. Public. 7, 12045 (2012). 